\title{
Towards wormhole beyond Horndeski
}

\author{
$S$. Mironov ${ }^{1,2, *}, V$. Rubakov ${ }^{1,3, * *}$, and $V$. Volkova ${ }^{1,3, * * *}$ \\ ${ }^{1}$ Institute for Nuclear Research of the Russian Academy of Sciences, 60th October Anniversary \\ Prospect, 7a, 117312 Moscow, Russia \\ ${ }^{2}$ Institute for Theoretical and Experimental Physics, Bolshaya Cheriomyshkinskaya, 25, 117218 \\ Moscow, Russia \\ ${ }^{3}$ Department of Particle Physics and Cosmology, Physics Faculty, M.V. Lomonosov Moscow State \\ University, Vorobjevy Gory, 119991 Moscow, Russia
}

\begin{abstract}
We address the issue of whether a no-go theorem for static, spherically symmetric wormholes, proven in Horndeski theories, can be circumvented by going beyond Horndeski. We show that the ghost instabilities which are at the heart of the no-go theorem, can indeed be avoided. The wormhole solutions with the latter property are, however, strongly fine tuned, and hence it is likely that they are unstable. Furthermore, it remains unclear whether these solutions have other pathologies, like gradient instabilities along angular and radial directions.
\end{abstract}

\section{Introduction and summary}

Horndeski theories [1] can violate the Null Energy Condition (NEC) in a healthy way. Their distinctive feature is that despite the presence of second derivatives in the Lagrangian, equations of motion are second order. Similar properties, but at the level of perturbations, hold in beyond Horndeski theory [2]. Because of potentially healthy NEC violation, Horndeski and beyond Horndeski theories have been recently explored for constructing various cosmological solutions, for instance, bounce and Genesis. However, there are no-go theorems [3-6] stating that classically stable, at all times, bouncing or Genesis solutions are absent in Horndeski theory. On the other hand, these theorems can be circumvented in beyond Horndeski theory, and a fully stable bouncing solution can be constructed [7-10].

Similar issues arise in the static, spherically symmetric context. The analog of the cosmological bounce in this case is static, traversable wormhole. One might expect, by analogy to cosmological setting, that there is a no-go theorem in Horndeski theory. This expectation is confirmed in Horndeski theory: static, spherically symmetric, asymptotically flat wormholes are plagued with ghost and/or radial gradient instability [11, 12]. By the same analogy, one might expect that the no-go theorem might be circumvented in beyond Horndeski theories, so that a fully stable wormhole might exist. On the other hand, censorship against the time machine and teleportatiton $[13,14]$ is also a weighty argument.

In this paper we address the latter issue, and concentrate on ghost and radial gradient instabilities. We find that, indeed, there exist wormholes without these instabilities in beyond

\footnotetext{
*e-mail: sa.mironov_1@physics.msu.ru

**e-mail: rubakov@inr.ac.ru

***e-mail: volkova.viktoriya@physics.msu.ru
} 
Horndeski theories. However, these solutions are strongly fine tuned. Our current understanding is that this fine tuning actually implies wormhole instability at non-linear level. Furthermore, we have not yet studied in full detail possible gradient and tachyon instabilities; although off hand there appears no reason for the existence of the latter, there might be surprizes here as well.

\section{NEC, bouncing Universe and wormhole}

The NEC is a fairly general property of matter degrees of freedom, which reads

$$
T_{\mu \nu} k^{\mu} k^{\nu} \geq 0
$$

where $k^{\mu}$ is an arbitrary null vector and $T_{\mu \nu}$ is energy-momentum tensor. The assumption that the NEC holds is crucial for Penrose theorem [15] that states that the presence of a trapped surface unavoidably leads to a singularity. In the cosmological context this means that contracting Universe ends up in a singularity and, by time reversal, that expanding Universe has a singularity in the past. Likewise, Penrose theorem and its generalizations forbid the existence of Lorentzian wormholes [16].

It is well-known that NEC violation in a healthy way requires very peculiar types of matter, see [17] for a review. For a long time there was even a belief that breaking NEC always leads to appearance of a pathological degree of freedom (under pathological degrees of freedom one usually means gradient instabilities and/or ghosts). The situation has changed fairly recently, when Horndeski theories (generalized Galileons) became a subject of intense study [18-21].

The (imperfect) analogy between bouncing Universe and static, spherically symmetric wormhole becomes clear once one writes (spatially flat) cosmological FLRW metric:

$$
d s^{2}=B(t) d t^{2}-a(t)^{2}\left(d r^{2}+r^{2}\left(d \theta^{2}+\sin ^{2} \theta d \varphi^{2}\right)\right),
$$

and static spherically symmetric metric:

$$
d s^{2}=A(r) d t^{2}-\frac{d r^{2}}{B(r)}-R(r)^{2}\left(d \theta^{2}+\sin ^{2} \theta d \varphi^{2}\right) .
$$

Modulo signs here and there, the only difference is that functions $A, B, R$ depend on radius but not time. The profiles of $a(t)$ and $R(r)$ for the bouncing Universe and wormhole, respectively, are quite similar (where on the right panel we think of $R \rightarrow+\infty$ as our Universe and $R \rightarrow-\infty$ as another Universe or very distant part of ours):
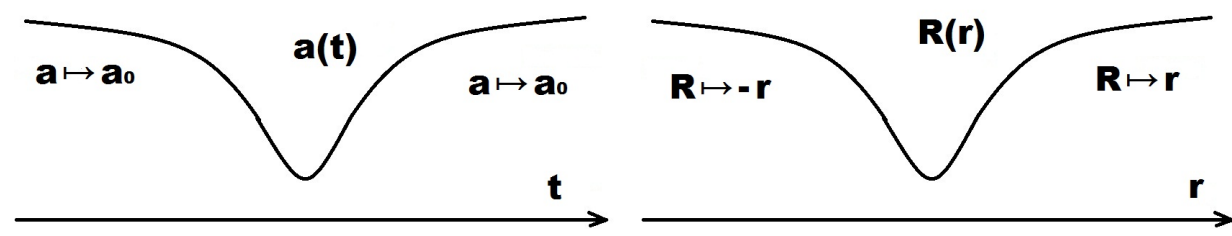
So, it is not surprising that theories that do not admit fully stable bouncing cosmologies, do not support stable static spherically symmetric wormholes as well, and vice versa. Gradient instabilities in bouncing cosmologies (wrong sign of spatial gradient terms in the quadratic action for small perturbations) have their counterparts as ghost instabilities of wormhole backgrounds (wrong sign of terms with time derivatives). This is precisely what happens in Horndeski theories (cf. Refs. [3, 4] for bouncing Universe and Refs. [11, 12] for wormholes). An intriguing question is whether the opposite is correct as well: beyond Horndeski theories do have completely stable bouncing solutions [7-10], do they support wormholes?

\section{Horndeski and beyond}

Horndeski theory is the most general scalar-tensor theory of gravity whose Lagrangian contains second derivatives, and yet the field equations are second order in derivatives. If one allows third derivatives in the equations of motion, but restricts the unconstrained equations for perturbations to have only second derivatives, then in the simplest cases one comes to beyond Horndeski Lagrangians. The most general forms of these Lagrangians are

$$
\begin{aligned}
& S=\int \mathrm{d}^{4} x \sqrt{-g}\left(\mathcal{L}_{2}+\mathcal{L}_{3}+\mathcal{L}_{4}+\mathcal{L}_{5}+\mathcal{L}_{\mathcal{B H}}\right), \\
& \mathcal{L}_{2}=F(\pi, X) \\
& \mathcal{L}_{3}=K(\pi, X) \square \pi \\
& \mathcal{L}_{4}=-G_{4}(\pi, X) R+2 G_{4 X}(\pi, X)\left[(\square \pi)^{2}-\pi_{; \mu v} \pi^{; \mu v}\right], \\
& \mathcal{L}_{5}=G_{5}(\pi, X) G^{\mu v} \pi_{; \mu \nu}+\frac{1}{3} G_{5 X}\left[(\square \pi)^{3}-3 \square \pi \pi_{; \mu v} \pi^{; \mu \nu}+2 \pi_{; \mu \nu} \pi^{; \mu \rho} \pi_{; \rho}^{\nu}\right], \\
& \mathcal{L}_{\mathcal{B H}}=\mathbf{F}_{\mathbf{4}}(\pi, X) \epsilon_{\sigma}^{\mu \nu \rho} \epsilon^{\mu^{\prime} v^{\prime} \rho^{\prime} \sigma} \pi_{, \mu} \pi_{, \mu^{\prime}} \pi_{; v v^{\prime}} \pi_{; \rho \rho^{\prime}} \\
& +\mathbf{F}_{\mathbf{5}}(\pi, X) \epsilon^{\mu \nu \rho \sigma} \epsilon^{\mu^{\prime} v^{\prime} \rho^{\prime} \sigma^{\prime}} \pi_{, \mu} \pi_{, \mu^{\prime}} \pi_{; \nu v^{\prime}} \pi_{; \rho \rho^{\prime}} \pi_{; \sigma \sigma^{\prime}}
\end{aligned}
$$

where $\pi$ is the scalar (Galileon) field, $X=g^{\mu v} \pi_{, \mu} \pi_{, v}, \pi_{, \mu}=\partial_{\mu} \pi, \pi_{; \mu v}=\nabla_{v} \nabla_{\mu} \pi, \square \pi=g^{\mu v} \nabla_{\nu} \nabla_{\mu} \pi$, $G_{4 X}=\partial G_{4} / \partial X$, etc. The Lagrangians $\mathcal{L}_{2}-\mathcal{L}_{5}$ give Horndeski theory, while $\mathcal{L}_{\mathcal{B H}}$ is characteristic of beyond Horndeski.

\section{No-go theorem}

In this section we briefly consider no-go theorem that forbids stable wormhole solutions in Horndeski theory [12]. Very similar theorem holds for spatially flat bouncing Universe in this theory. We give the following lemma first. Suppose we have a "nice" function $f(x)$ defined for all $x$ from $-\infty$ to $\infty$. Now, if $f^{\prime}(x)>\epsilon>0$, then $f\left(x_{0}\right)=0$ at some point $x_{0}$. Let us illustrate this in the following plots:

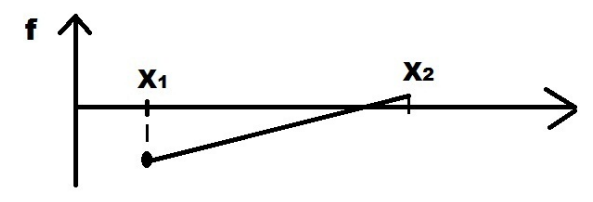

If $f\left(x_{1}\right)<0, \quad$ then $f\left(x_{2}\right) \geq 0$, where $x_{2}=x_{1}-\frac{f\left(x_{1}\right)}{\epsilon}$. 


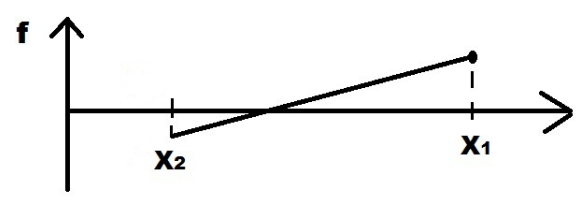

Alternatively, if $f\left(x_{1}\right)>0, \quad$ then $f\left(x_{2}\right) \leq 0$, where $x_{2}=x_{1}-\frac{f\left(x_{1}\right)}{\epsilon}$.

In fact, the function $f(x)$ may be singular at one or more points, as shown below:

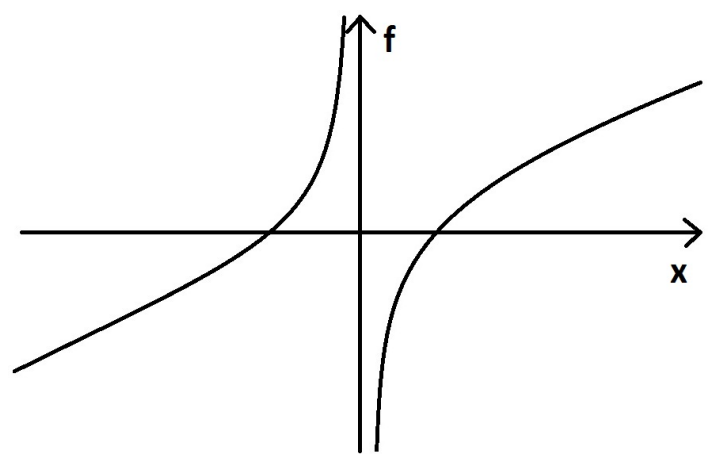

Having this in mind, we consider perturbations of metric and scalar field in the spherically symmetric background (3). We begin with Horndeski theory, $\mathbf{F}_{\mathbf{4}}(\pi, X)=\mathbf{F}_{\mathbf{5}}(\pi, X)=0$. quadratic Lagrangian for these perturbations has been derived by Kobayashi, Motohashi and Suyama [22, 23] who make use of Regge-Wheeler approach. In the spherically symmetric background, perturbations are classified into parity even and parity odd sectors. There are two fields $v^{i}(i=1,2)$ in parity even sector, and their Lagrangian is

$$
\mathcal{L}_{\text {even }}=\frac{1}{2} \mathcal{K}_{i j} \dot{v}^{i} \dot{v}^{j}-\frac{1}{2} \mathcal{P}_{i j} v^{i^{\prime}} v^{j^{\prime}}-Q_{i j} v^{i} v^{j^{\prime}}-\frac{1}{2} \mathcal{M}_{i j} v^{i} v^{j},
$$

where the coefficients $\mathcal{K}_{i j}(r), \mathcal{P}_{i j}(r), Q_{i j}(r), \mathcal{M}_{i j}(r)$ are expressed through the functions in the Horndeski Lagrangian evaluated on the spherically symmetric background, and prime denotes $\partial / \partial r$. Expression (5) is written for spherical harmonics $v_{l m}^{i}$, and the functions $\mathcal{K}_{i j}(r)$, etc., depend also on angular momentum $l$.

As we already pointed out, we concentrate on ghost and radial gradient instability issue. The absence of these instabilities in parity even sector requires

$$
\mathcal{K}_{11}>0, \quad \operatorname{det}(\mathcal{K})>0, \quad \mathcal{P}_{11}>0, \quad \operatorname{det}(\mathcal{P})>0
$$

(the absence of gradient instabilities in angular directions, which we do not consider, requires similar relations for dominant terms with dependence on $l$ ).

For the odd parity modes the quadratic Lagrangian reads

$$
\mathcal{L}_{\text {odd }}=\frac{l(l+1)}{2(l-1)(l+2)} \sqrt{\frac{B}{A}} R^{2}\left[\frac{\mathcal{H}^{2}}{\sqrt{A} \mathcal{G}} \dot{Q}^{2}-\frac{\sqrt{B} \mathcal{H}^{2}}{\mathcal{F}}\left(Q^{\prime}\right)^{2}-\frac{l(l+1)}{R^{2}} \cdot \mathcal{H} Q^{2}-V(r) Q^{2}\right],
$$

so we have similar restrictions, the relevant ones for the no-go theorem being

$$
\mathcal{F}>0, \quad \mathcal{H}>0,
$$

where, again, $\mathcal{F}(r)$ and $\mathcal{H}(r)$ are expressed through the Lagrangian functions evaluated on the spherically symmetric background Also, background metric coefficients cannot cross zero: 


$$
A>0, B>0, R>0 .
$$

Now, the key point is that modulo a manifestly positive factor, det $K$ reads

$$
\operatorname{det} K \sim \mathcal{F}\left(2 \xi^{\prime}-\mathcal{F}\right)>0,
$$

where $\xi(r)$ has the following structure

$$
\xi=\left[\frac{(R \mathcal{H})^{2}}{\Theta}\right],
$$

with

$$
\begin{aligned}
\mathcal{H} & =2 G_{4}+2 G_{4 X} B \pi^{\prime 2}, \\
\Theta & =2 \mathcal{H} R R^{\prime}+\Xi \pi^{\prime},
\end{aligned}
$$

where

$$
\Xi=-2 G_{4 \pi} R^{2}-4 G_{4 X} B R R^{\prime} \pi^{\prime}+2 G_{4 \pi X} B R^{2}\left(\pi^{\prime}\right)^{2}+4 G_{4 X X} B^{2} R R^{\prime}\left(\pi^{\prime}\right)^{3},
$$

and we take $G_{5}(\pi, X)=0$ for the sake of brevity. Equations (9), (10) enable one to prove the no-go theorem $[5,12]$. Indeed, in full analogy with the homogeneous case in Refs. $[4,10], \xi$ cannot cross zero, but it has to, since its derivative is always positive (see the lemma above with $\xi$ being a counterpart to function $f(x)$ ).

\section{What becomes of no-go theorem for wormholes beyond Horndeski}

The no-go theorem does not work for wormholes beyond Horndeski theory. In spherically symmetric background (3), like in the cosmological case [4, 10], both relations (9) and (10) get modified. Namely, the analog of (10) reads

$$
\xi=\left[\frac{R^{2} \mathcal{H}(\mathcal{H}-\mathcal{D})}{\Theta}\right],
$$

where the expressions for $\mathcal{H}$ and $\Theta$ are now (we set $G_{5}=\mathbf{F}_{\mathbf{5}}=0$ in what follows)

$$
\begin{aligned}
\mathcal{H} & =2 G_{4}+2 G_{4 X} B \pi^{\prime 2}+2 \mathbf{F}_{4} B^{2} \pi^{\prime 4}, \\
\Theta & =2 \mathcal{H} R R^{\prime}+\Xi \pi^{\prime},
\end{aligned}
$$

with

$$
\begin{aligned}
\Xi= & -2 G_{4 \pi} R^{2}-4 G_{4 X} B R R^{\prime} \pi^{\prime}+2 G_{4 \pi X} B R^{2}\left(\pi^{\prime}\right)^{2}+4 G_{4 X X} B^{2} R R^{\prime}\left(\pi^{\prime}\right)^{3} \\
& -16 \mathbf{F}_{4} B^{2} R R^{\prime}\left(\pi^{\prime}\right)^{3}+4 \mathbf{F}_{4 \mathbf{X}} B^{3} R R^{\prime}\left(\pi^{\prime}\right)^{5},
\end{aligned}
$$

while

$$
\mathcal{D}=2 \mathbf{F}_{4} B^{2} \pi^{\prime 4}
$$

The requirement that $\operatorname{det} K>0$ is now

$$
\operatorname{det} K \sim(\mathcal{F}-C Q)\left(2 \xi^{\prime}-\mathcal{F}\right)-C Q^{2}>0,
$$

where

$$
Q=a_{1} \cdot \frac{\left(a_{2} \mathcal{D}\right)^{\prime}}{R^{\prime}}, \quad C=\frac{4 l(l+1)}{(l+2)(l-1)},
$$


and both $a_{1}$ and $a_{2}$ are manifestly positive: $a_{2}=a_{1} \cdot R / 2=\sqrt{A} /\left(\pi^{\prime} \sqrt{B}\right)$. Assuming that asymptotically, as $r \rightarrow \pm \infty$, the theory reduces to GR, we have $Q \rightarrow 0$, so that $\mathcal{F}-Q>0$ as $r \rightarrow \pm \infty$. Positivity of $\operatorname{det} K$ requires that $\mathcal{F}-Q$ does not change sign, so we still have to require that $\xi^{\prime}>\frac{1}{2} \mathcal{F}>0$ at all $r$. However, again in full analogy with the cosmological case $[4,10], \xi$ can safely cross zero in beyond Horndeski theory due to the additional term $\mathcal{D}$, so the no-go theorem no longer works.

An explicit example of such a solution is shown in Fig. 1 (for the sake of brevity we do not present explicitly all Lagrangian functions, but they can be chosen in a smooth way with all EOM obeyed). In our set up $R=\sqrt{1+r^{2}}$ while $A=B=1$ in eq. (3). For the wormhole construction we make use of functions $F, G_{4}$ and $F_{4}$ only. The solution is arranged so that far away from the wormhole, i.e. at $r \rightarrow \pm \infty$,

$$
F(\pi, X) \rightarrow 0, \quad G_{4}(\pi, X) \rightarrow 1 / 2, \quad \mathbf{F}_{4}(\pi, X) \rightarrow \text { const },
$$

hence, both asymptotic states are still described by beyond Horndeski theory. Our choice of the Lagrangian functions ensures that $\mathcal{F}, \mathcal{H}, \mathcal{G}, \mathcal{K}_{11}$ and $\operatorname{det}(\mathcal{K})$ are strictly positive and corresponding sound speed squared is subluminal. Thus, the solution appears to be ghost-free in the parity even sector and free of both gradient and ghost instabilities in the odd sector.

However, the great disadvantage of this solution is strong finetuning. One has to make $\mathcal{D}^{\prime}=0$ at the exact same point, where $R^{\prime}=0$ (the wormhole throat), otherwise $Q$ is singular and $\operatorname{det} K>0$ constraint is not satisfied. It indicates an unhealthy nature of such setup: any infinitesimal deviation from this solution results in ghost appearance.

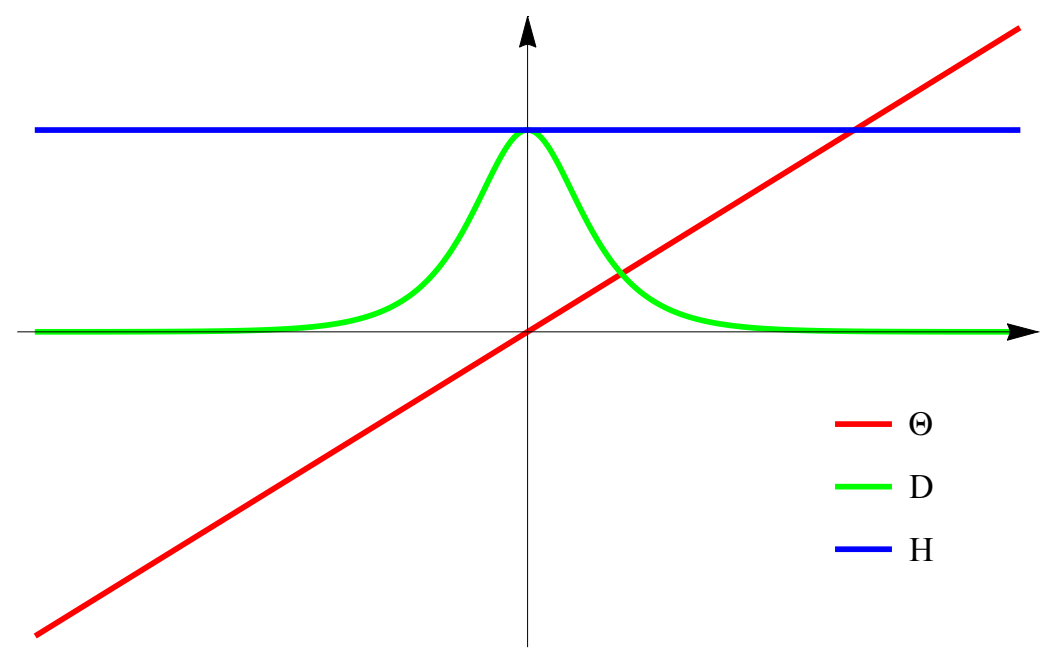

Figure 1. $\Theta$ is a linear function of radius, $\mathcal{H}$ is a constant and $\mathcal{D}=\cosh ^{-2}(r)$

\section{Acknowledgements}

This work has been supported by Russian Science Foundation grant 14-22-00161.

\section{References}

[1] G. Horndeski, Int. J. Theor. Phys. 10, 363 (1974) 
[2] J. Gleyzes, D. Langlois, F. Piazza and F. Vernizzi, Phys. Rev. Lett. 114, 211101 (2015)

[3] M. Libanov, S. Mironov, V. Rubakov, JCAP 1608, 037 (2016)

[4] T. Kobayashi, Phys. Rev. D 94, 043511 (2016)

[5] R. Kolevatov, S. Mironov, Phys. Rev. D 94, 123516 (2016)

[6] S. Akama, T. Kobayashi, Phys. Rev. D 95, 064011 (2017)

[7] Y. Cai, Y. Wan, H. Li, T. Qiu, Y. Piao, JHEP 1701, 090 (2017)

[8] P. Creminelli, et al, JCAP 1611, 047 (2016)

[9] Y. Cai, Y. Piao, JHEP 1709, 027 (2017)

[10] R. Kolevatov, S. Mironov, N. Sukhov, V. Volkova, JCAP 1708, 038 (2017)

[11] V. Rubakov, Theor.Math.Phys. 188, 1253-1258 (2016), Teor.Mat.Fiz. 188, 337-342 (2016)

[12] O. Evseev, O. Melichev, Phys. Rev. D 97, 124040 (2018)

[13] M. S. Morris and K. S. Thorne, Am. J. Phys. 56, 395 (1988).

[14] M. S. Morris, K. S. Thorne and U. Yurtsever, Phys. Rev. Lett. 61, 1446 (1988).

[15] R. Penrose, Phys. Rev. Lett. 14, 57 (1965)

[16] D. Hochberg and M. Visser, Phys. Rev. D 58, 044021 (1998)

[17] V. Rubakov, Phys.Usp. 57, 128-142 (2014), Usp.Fiz.Nauk 184, 137-152 (2014)

[18] M. A. Luty, M. Porrati and R. Rattazzi, JHEP 0309, 029 (2003).

[19] A. Nicolis and R. Rattazzi, JHEP 0406, 059 (2004).

[20] A. Nicolis, R. Rattazzi and E. Trincherini, Phys. Rev. D 79, 064036 (2009).

[21] P. Creminelli, A. Nicolis and E. Trincherini, JCAP 1011, 021 (2010) [arXiv:1007.0027 [hep-th]].

[22] T. Kobayashi, H. Motohashi, T. Suyama, Phys. Rev. D 85, 084025 (2012), Erratum: Phys.rev. D 96 no.10, 109903 (2017)

[23] T. Kobayashi, H. Motohashi, T. Suyama, Phys. Rev. D 89, 084042 (2014) 